\title{
Exploración psicológica de madres adolescentes con parejas mayores que ellas ${ }^{a}$.
}

\author{
Monserrat Muñoz ${ }^{1, *}$, Rubén A .Díaz H. ${ }^{2}$ \\ ${ }^{1}$ Licenciada en Psicología, Egresada de la Universidad Católica Santa María La Antigua (USMA), Panamá, \\ República de Panamá. \\ ${ }^{2}$ Profesor, Escuela de Psicología, Universidad Católica Santa María La Antigua (USMA), Panamá, República \\ de Panamá. \\ *Autor para correspondencia. Email: mdanielamunoz@hotmail.com
}

Recibido: 10 de agosto de 2015

Aceptado: 14 de agosto de 2015

\begin{abstract}
This study aims to identify a profile of a group of teenage mothers $(n=15)$ in Panama City, who are in a sentimental relationship with partners who are 5 or more years older than them. Participants were evaluated with the Adult Attachment Questionnaire (Melero \& Cantero, 2008). In addition, semistructured interviews were conducted in order to define a socio psychological profile and to determine the perceptions about other's opinions of their pregnancy and their relationship. Results show an insecure pattern of attachment in most participants. Teenagers perceive negative opinions from their families toward their pregnancy and their relationship. Most of these teenagers chose not to use contraceptive methods despite of knowing its benefits. Teenagers are not mature enough for decisionmaking, thus, they find themselves wrapped in social, emotional and economic risks.
\end{abstract}

Keywords: Attachment, Self-esteem, Teenage pregnancy, Teenage dating, Contraceptive methods.

\section{Resumen}

Esta investigación tiene como objetivo identificar un perfil de un grupo de madres adolescentes ( $\mathrm{n}=15)$ de la Ciudad de Panamá, cuyas parejas sentimentales son por lo menos cinco años mayor que ellas. Las participantes fueron evaluadas con el Cuestionario de Apego Adulto (Melero \& Cantero, 2008). Adicionalmente, se realizaron entrevistas semiestructuradas para determinar su perfil socio-psicológico y las percepciones que tienen acerca de las opiniones de otros con respecto a su embarazo y a su

\footnotetext{
${ }^{a}$ Esta investigación presenta un análisis parcial de los resultados presentados en "Patrones de apego y perfil socio-psicológico de madres adolescentes y adolescentes embarazadas de 14 a 19 años de edad cuyas parejas son por lo menos cinco años mayores, en la ciudad de Panamá", tesis de Licenciatura en Psicología presentada en la USMA por Monserrat Muñoz en 2015 .
} 
relación. Los resultados muestran un patrón de apego inseguro en la mayoría de las participantes. Las adolescentes perciben mayormente que su familia tiene opiniones negativas sobre su embarazo y su relación de pareja. La mayoría de estas adolescentes eligieron no utilizar métodos anticonceptivos a pesar de conocer sus beneficios. Se concluye que las adolescentes no han desarrollado las características necesarias para su maduración y toma de decisiones por lo que se han visto envueltas en situaciones de riesgo social, de salud y económicos.

Palabras clave: Apego, Autoestima, Embarazo adolescente, Noviazgo adolescente, Anticonceptivos.

\section{Introducción.}

El embarazo adolescente es un problema de índole social, cultural, ambiental y económico (Organización Mundial de la Salud [OMS], 2009, 2012). Las cifras estadísticas en Panamá reflejan que, para el año 2010, existían 22,503 madres entre 15 y 19 años, lo que representa un 15.4\% de la población de dicho grupo de edad (Instituto Nacional de Estadística y Censo [INEC], 2010). Por otra parte, alrededor de $46.9 \%$ de las mujeres con edades entre 15 y 19 años mantienen relaciones de pareja con personas que son por lo menos cinco años mayores que ellas (INEC, 2014).

Diversos factores sociales, culturales y psicológicos han sido relacionados con el embarazo adolescente: menor escolaridad, menor apoyo social, mayor tasa de mortalidad a la hora de dar a luz y dificultades económicas (De León y otros, 2011; OMS, 2009, 2012; Programas de las Naciones Unidas para el Desarrollo [PNUD], 2014).

La Ley N²9 del 13 de junio del 2001 garantiza la salud y la educación de la adolescente embarazada en Panamá. Sin embargo, la mayoría de las adolescentes abandonan la escuela después de haber dado a luz a su primer hijo (Austin y otros, 2000; PNUD, 2014, Varela, 2010).

La disfuncionalidad familiar también ha sido identificada como un factor de riesgo con respecto al embarazo adolescente (Manlove, Terry-Humen e Ikramullah, 2006; Rangel, Valerio, Patiño y García, 2004). Estudios realizados en Panamá señalan que las adolescentes embarazadas provienen de un hogar desintegrado y que la mayoría de ellas abandona la su familia de origen (Austin y otros, 2000). Sin embargo, estadísticas más recientes revelan que actualmente la mayoría de las adolescentes permanecían en casa de su familia de origen después de haber dado a luz (Varela, 2010).

Una revisión bibliográfica realizada de 1988 a 1998 sobre el perfil de salud sexual y reproductiva de los y las adolescentes y jóvenes de América Latina y el Caribe (Camacho Hubner, 2000) indica que las adolescentes que empiezan una vida de pareja a edad temprana tienen mayor porcentaje de quedar embarazadas y tienen mayor probabilidad de abandonar sus estudios. Además, se encontró que la mayor parte de adolescentes que estuvieron casadas o tuvieron una relación significativa la hicieron con un hombre por lo menos tres años mayor a ella.

Las adolescentes cuyas parejas tienen edad considerablemente mayor que ellas son más propensas a tener relaciones sexuales sin protección lo que puede deberse a la pérdida de poder en la relación que 
Invest. pens. crit.

Vol. 3, No. 2, mayo-agosto 2015

pp. $20-31$

produce una disparidad de género y se manifiesta en ceder a las peticiones de la pareja (Canto, 2012; Manlove y otros, 2006; Miller, Clark y Moore, 2013).

Por otra parte la maternidad adolescente no planeada está asociada con violencia en la pareja y mayor propensión al aborto (Kaye, Mirembe, Bantebya, Johansson y Ekstrom, 2006; Silverman, Gupta, Decker y Raj, 2007; Stephenson, Koeing, Acharya y Roy, 2008), elevados niveles de estrés y tensión en la pareja y mayor probabilidad de que ésta se disuelva durante el primer año después del nacimiento del niño (Hohmann-Marriot, 2009). Esto es importante porque una mala relación de pareja incide en la capacidad de la madre para ofrecer a su hijo un ambiente psicológico óptimo para su desarrollo emocional (Winnicott, 1960).

En Panamá se han realizado estudios en torno al tema del embarazo adolescente y sobre madres adolescentes dirigidos principalmente a la caracterización sociodemográfica del problema. Sin embargo, los procesos psicológicos implicados en el desarrollo y calidad de vida de esta población no han sido suficientemente abordados.

Este estudio está centrado en establecer un perfil de los patrones de apego en madres adolescentes cuyas parejas sean 5 o más años mayores con el fin de conocer con mayor detalle los procesos psicológicos involucrados en esta población, contribuyendo a establecer evidencias sobre adolescentes proclives a incidir en una situación como ésta y permitiendo, en forma oportuna, realizar las intervenciones pertinentes.

\section{Materiales y métodos.}

\section{$\underline{\text { Participantes }}$}

Quince madres adolescentes que acudieron al Servicio de Ginecología y Pediatría del Centro de Salud de Veranillo entre los meses de febrero y marzo, fueron invitadas y aceptaron participar en el estudio. La selección se llevó a cabo mediante una técnica de muestreo accidental, utilizando como criterios de inclusión madre adolescente, tener una pareja sentimental por lo menos 5 años mayor que ella, que la relación de pareja tenga una duración de por lo menos dos veces. La edad de las participantes estaba en el rango entre 14 y 19 años (mediana $=17$ años, desviación absoluta mediana $=1$ año).

\section{$\underline{\text { Materiales }}$}

Cuestionario de apego adulto (CAA). Es un instrumento de auto-reporte de 40 preguntas diseñado por Melero y Cantero, (2008), con el objetivo de establecer los estilos afectivos en adultos españoles. Se compone de cuatro escalas: Baja autoestima, necesidad de aprobación y miedo al rechazo; Resolución hostil de conflictos, rencor y posesividad; Expresión de sentimientos y comodidad con las relaciones y Autosuficiencia emocional e incomodidad con la intimidad que permiten identificar cuatro estilos de apego: seguro, huidizo temeroso, preocupado y huidizo alejado. Consiste de 40 reactivos, con una escala de respuesta tipo Likert de 5 puntos y ofrece un índice para cada uno de las cuatro escalas 
mencionadas. Los autores de la prueba reportan que las escalas tienen índices de consistencia interna aceptables y evidencia de validez factorial.

Cuestionario de datos generales (CDG). Cuestionario diseñado para los fines de este estudio que recoge información sobre los datos generales de la adolescente, estatus económico, escolaridad, lugar de nacimiento, lugar residencia, estado civil y edad de la pareja.

Entrevista estructurada. Entrevista diseñada para los fines de este estudio que aborda los temas sobre la percepción que tienen las adolescentes sobre su embarazo, maternidad y relación de pareja. Al mismo tiempo se busca medir la percepción sobre la aceptación de sus familiares, pares y sociedad.

\section{$\underline{\text { Procedimiento }}$}

Luego de obtener aceptación voluntaria para participar en el estudio, las madres adolescentes fueron evaluadas mediante la aplicación de dos cuestionarios de auto-reporte (CAA y CDG). Los cuestionarios fueron respondidos en el consultorio médico en donde recibieron la consulta de servicio de Pediatría o Ginecología por la que habían concurrido al Centro de Salud. Los resultados numéricos de cada una de las dimensiones de la prueba CAA se analizaron mediante estadísticas descriptivas. Para todos los casos se eligió la mediana como medida de tendencia central y la desviación absoluta mediana como medida de dispersión en vista de que no se cumplió con el requisito de aproximación a la distribución normal: todas las escalas poseen una distribución unimodal pero dos de ellas presentan una asimetría significativa.

Las respuestas de las adolescentes a la entrevista estructurada fueron codificadas aplicando la técnica de análisis de contenido de resumen descrita por Mayring (2014). Las categorías generadas se analizaron mediante estadísticas descriptivas de frecuencia.

\section{Resultados}

Características generales: educación, familia y uso de métodos anticonceptivos.

La Tabla 1 resume los resultados del cuestionario de datos generales con respecto a la educación, la familia de origen y la planificación del embarazo por parte de las adolescentes de la muestra.

El embarazo precoz tuvo un impacto negativo en la continuidad de los estudios. La mayoría de las adolescentes (63.3\%) abandonó la escuela secundaria. Tres de las adolescentes que no abandonaron la escuela ya se graduaron de bachiller y dos continúan sus estudios por módulos.

Con respecto a las familias de origen de las madres adolescentes, la mayoría (60.0\%) creció en familias con padres separados. En este punto también es destacable la posible existencia de un patrón familiar en el embarazo precoz: en todos los casos estudiados el primer embarazo de las madres de las adolescentes ocurrió entre los 15 y 23 años. 
Invest. pens. crit.

Vol. 3, No. 2, mayo-agosto 2015

pp. $20-31$

Tabla 1.

Educación, familia y anticoncepción.

\begin{tabular}{lcc}
\hline Deserción escolar & $\mathbf{f}$ & $\mathbf{0}$ \\
\hline Sí & 10 & 66.7 \\
No & 5 & 33.3 \\
Padres separados & & \\
Sí & 9 & 60.0 \\
No & 6 & 40.0 \\
Conocimiento de métodos anticonceptivos & & \\
Sí & 14 & 93.3 \\
No & 1 & 7.7 \\
Uso de métodos anticonceptivos & & \\
\hline Sí & 0 & 0.0 \\
No & 15 & 100.0 \\
\hline Porchajes (o).
\end{tabular}

Notas: Frecuencia (f) y Porcentajes (\%).

Catorce adolescentes manifestaron conocer los beneficios del uso de métodos anticonceptivos, especialmente del condón. Sin embargo, ninguna de ellas lo utiliza, alegando como justificación más frecuente el hecho de que no les gusta. Otras justificaciones que aparecieron en las respuestas para no utilizar métodos anticonceptivos tienen que ver con la creencia de que en una relación estable no debe utilizarse, o que a la pareja le aseguró que no pasaría nada. Es importante notar que en dos de los casos, las madres adolescentes aseguraron que el embarazo fue planeado; ellas mantienen relaciones de pareja desde hace más de 3 años con hombres de 30 y 36 años.

\section{Apego.}

En general, el grupo de participantes presentó puntuaciones altas en dos de las dimensiones que suelen estar presentes en personas con patrones de apego inseguro, especialmente las que están relacionadas con los estilos de apego alejado y temeroso-hostil (Tabla 2). También se halla la presencia, en un nivel moderado, de indicadores de baja autoestima, necesidad de aprobación y miedo al rechazo, todos ellos relacionados con el estilo de apego preocupado. Por último, la mediana de puntuaciones en la escala relacionada con un estilo de apego seguro corresponde con un nivel Bajo.

Tabla 2.

Puntuaciones obtenidas en las dimensiones de la prueba $C A A$.

\begin{tabular}{|c|c|c|c|}
\hline \multirow[b]{2}{*}{ Dimensión } & \multicolumn{2}{|c|}{ Estadísticos descriptivos } & \multirow[b]{2}{*}{ Valoración ${ }^{a}$} \\
\hline & Mdn & DAM & \\
\hline $\begin{array}{l}\text { E1: Baja autoestima, necesidad de aproba- } \\
\text { ción y miedo al rechazo }\end{array}$ & 39 & 6 & Moderado \\
\hline $\begin{array}{l}\text { E2: Resolución hostil de conflictos, rencor } \\
\text { y posesividad }\end{array}$ & 37 & 7 & Alto \\
\hline $\begin{array}{l}\text { E3: Expresión de sentimientos y comodi- } \\
\text { dad con las relaciones }\end{array}$ & 36 & 4 & Bajo \\
\hline $\begin{array}{l}\text { E4: Autosuficiencia emocional e incomodi- } \\
\text { dad con la intimidad }\end{array}$ & 23 & 4 & Alto \\
\hline
\end{tabular}


Invest. pens. crit.

Vol. 3, No. 2, mayo-agosto 2015

pp. $20-31$

Si bien el patrón de resultados en las cuatro dimensiones no se ajusta a ninguno de los cuatro tipos de apego reportados por Melero y Cantero (2008), sí pueden orientar a describir el estilo de apego de estas adolescentes como de tipo inseguro.

Percepción de las madres adolescentes sobre las opiniones de familiares y amigos con respecto a su embarazo

Tabla 3.

Percepción de las opiniones de los otros con respecto a su embarazo

\begin{tabular}{lcccc}
\hline \multicolumn{1}{c}{ Preguntas } & Positivo & Negativo & Mixto & Desconoce \\
\hline $\begin{array}{l}\text { 1. ¿Cómo tú te sientes con respecto a ser } \\
\text { madre? }\end{array}$ & $12(80.0)$ & $1(6.7)$ & $2(13.3)$ & $0(0.0)$ \\
$\begin{array}{l}\text { 2. ¿Cómo crees que tus familiares se sien- } \\
\text { ten con respecto a tu embarazo? ¿Qué } \\
\text { crees que tus familiares piensan con res- } \\
\text { pecto a tu embarazo? }\end{array}$ & & & \\
& & & & \\
$\begin{array}{l}\text { 3. ¿Qué crees que piensan tus amigos de tu } \\
\text { embarazo? ¿Cómo crees que se sienten con } \\
\text { respecto a tu embarazo? }\end{array}$ & $5(33.3)$ & $3(60.0)$ & & \\
\end{tabular}

Notas: Los valores fuera de los paréntesis corresponden a la frecuencia de sujetos cuyas respuestas fueron incluidas en la categoría correspondiente; los valores dentro de los paréntesis, a los porcentajes.

Sentimientos relacionados con la maternidad. La mayoría de las adolescentes manifestaron sentimientos positivos con respecto a la maternidad, argumentando que consideraban a su hijo como "una bendición" o que "ser mamá no cambia nada".

Dos adolescentes manifestaron sentimientos mixtos, como "es difícil, pero me siento bien" o "estoy contenta en parte, y por otra no, por la edad".

La adolescente que expresó sentimientos negativos hizo referencia a la consideración de que el embarazo le generó angustia sobre cómo continuaría la escuela. En efecto, ella fue una de las adolescentes que desertó de la educación secundaria.

Percepción de las opiniones de la familia. Como se observa en la Tabla 3 la mayoría de las adolescentes perciben que sus familiares tienen sentimientos y pensamientos negativos con respecto a su embarazo. Las respuestas reflejan la percepción de que los familiares recibieron la noticia con tristeza, vergüenza e ira. Los sentimientos negativos de los familiares percibidos por las adolescentes eran atribuidos a la edad en que se produjo el embarazo más que al embarazo en sí. En las respuestas de las adolescentes 
Invest. pens. crit.

Vol. 3, No. 2, mayo-agosto 2015

pp. 20-31

también aparece la preocupación de los padres acerca de cómo el embarazo se convierte en un obstáculo para continuar la educación de las jóvenes. Por su parte, ellas expresaron sentir miedo al contarlo a sus padres porque se sentían solas o esperaban que las regañarían.

Una menor cantidad de adolescentes perciben que sus familiares tienen sentimientos y pensamientos mixtos con relación a su embarazo. Se destaca que, en ocasiones, esta ambigüedad en la respuesta de los familiares tenía que ver con discordancia entre las respuestas de los padres: uno apoya el embarazo mientras que el otro no.

Solamente 2 adolescentes registraron haber percibido pensamientos positivos por parte de sus familiares, señalando que "fue una fiesta en su casa" y que los padres estaban felices porque "iban a tener un nieto.

Percepción de las opiniones de las amistades. La mayoría de las adolescentes manifiesta desconocer qué sienten o piensan sus amistades con respecto a su embarazo. Tres de estas adolescentes indicaron que no tenían amigos.

Otro grupo de las adolescentes perciben que sus amigos se sintieron felices y contentos con su embarazo. En menor medida, aparecen respuestas que señalan que los amigos se sintieron molestos por el evento.

Percepción de las madres adolescentes sobre las opiniones de familiares y amigos con respecto a su relación de pareja.

Las percepciones de las adolescentes sobre las opiniones de familiares y amigos con respecto a su relación de pareja se registran en la Tabla 4.

\begin{tabular}{|c|c|c|c|c|}
\hline Preguntas & Positivo & Negativo & Mixto & Desconoce \\
\hline $\begin{array}{l}\text { 1. ¿Cómo te sientes con tu rela- } \\
\text { ción de pareja? }\end{array}$ & $14(93.3)$ & $1(7.3)$ & $0(0.0)$ & $0(0.0)$ \\
\hline $\begin{array}{l}\text { 2. ¿Tus padres aprueban tu rela- } \\
\text { ción? ¿Por qué? }\end{array}$ & $5(33.3)$ & $4(26.7)$ & $3(20.0)$ & $1(7.3)$ \\
\hline $\begin{array}{l}\text { 3. ¿Crees que tus amigos aprueban } \\
\text { tu relación? ¿Por qué? }\end{array}$ & $6(40.0)$ & $2(13.3)$ & $0(0.0)$ & $7(46.7)$ \\
\hline
\end{tabular}

Bienestar en la relación de pareja. La mayoría de las participantes expresaron sentirse bien en su relación de pareja. Este sentimiento de bienestar estaba relacionado en su mayoría con la participación de la pareja en las tareas de la casa y la relación que mantenía la misma con su familia. Solamente una 
Invest. pens. crit.

Vol. 3, No. 2, mayo-agosto 2015

pp. 20-31

adolescente manifestó sentirse insatisfecha con su relación, argumentando que no se entienden como pareja.

Aprobación de la relación por los padres. Cinco adolescentes manifestaron que sus padres aceptaban la relación. Sin embargo, solamente dos de ellas pudieron identificar una razón para esta creencia ("[solo] le importa que me trate bien”, “[cree] que él es responsable”). Las otras tres adolescentes que perciben que sus padres aceptan la relación señalan que creen eso porque no les han dicho lo contrario. Una de estas últimas manifestó que cree que sus padres perciben a su pareja como muy controlador. Esta es la adolescente que manifiesta que su pareja es muy controlador.

Cuatro adolescentes perciben que sus padres no aceptan la relación de pareja y atribuyen a los padres sentimientos fuertes en contra de la pareja ("mi mamá lo quiere matar", "lo odian"). Otras respuestas indican que las preocupaciones de los padres se centran en el desbalance de poder producto de la edad (“piensan que me puede presionar"), en que sostengan relaciones sexuales y en la diferencia de edad.

Percepción de aprobación de la relación por parte de sus amistades. La mayoría de las adolescentes perciben que sus amigos aceptan su relación porque se llevan bien con su pareja. Una de las adolescentes de este grupo percibe que sus amigos creen que su pareja "es muy viejo".

Las dos que perciben opiniones negativas, argumentan que sus amigos se llevan mal con la pareja o porque la pareja es "un vago y un bobo" que "no terminó la escuela".

\section{Discusión.}

En esta investigación se exploró el apego de un grupo de madres adolescentes y las percepciones que estas tenían sobre las opiniones de sus familiares y amigos con respecto a su embarazo y a su relación de con una pareja por lo menos cinco años mayor que ellas.

De acuerdo con los resultados de la prueba CAA, el grupo de madres adolescentes evaluado presenta elevados indicadores tanto de Resolución hostil de conflictos, rencor y posesividad, como de Autosuficiencia emocional e incomodidad con la intimidad. Podemos encontrar que estos rasgos van de acuerdo con el estilo de Apego Desvalorizador propuesto por Bartholomew y Horowitz (1991), donde el modo de relacionarse de las adolescentes es determinado por la desconfianza y rechazo al establecer relaciones.

Los patrones de apego se desarrollan con base en las experiencias que el infante tiene con sus figuras de apego primarias. Si se desarrollan vínculos inestables estos se verán reflejados en la forma de relacionarse durante la adolescencia y la adultez. La mayoría las adolescentes entrevistadas reflejan haber crecido en familias desintegradas e inestables por lo que es posible asumir que esta condición puede estar relacionada con los patrones de apego encontrados en la prueba (Temeroso- Hostil y Alejado). 
Invest. pens. crit.

Vol. 3, No. 2, mayo-agosto 2015

pp. $20-31$

Distintos estudios indican que el embarazo adolescente está vinculado con disfuncionalidad familiar donde la gran mayoría de las adolescentes encuestadas se encontraban en un núcleo familiar desintegrado; hay antecedentes de embarazos precoces en madres y abuelas de las adolescentes (Cueva, Olvera y Chumacera, 2005; Manlove y otros, 2006; Rangel y otros, 2004). Este estudio reveló que existe un patrón familiar relacionado con el embarazo en las adolescentes ya que todos los casos entrevistados tienen antecedentes de este tipo de embarazo en su familia donde la mayoría de ellas crecieron en familias con padres divorciados o separados.

Los patrones de apego encontrados también pueden estar asociados a que las adolescentes encuestadas viven en situación de riesgo social. Este planteamiento coincide con los datos obtenidos por investigaciones recientes realizadas en países como Canadá y Estados Unidos (Cyr, Euser, Bakermans-Kranenburg y Van Ljzendoorn, 2010; Peterson, Buser y Westburg, 2010). Los adolescentes que crecen en este tipo de entornos son más propensos a verse involucrados en conductas de alto riesgo lo que encaja con las conductas de las adolescentes al involucrarse en relaciones con hombres mayores y elegir no utilizar anticonceptivos en sus relaciones sexuales.

Los resultados de esta investigación también indicaron que gran número de adolescentes conocían los beneficios de utilizar anticonceptivos y aun así decidieron no utilizarlos por diversas razones. Los datos estadísticos en Panamá (De León y otros, 2011; INEC, 2014) reflejan que aproximadamente entre 65\% y 85\% de las mujeres de entre 14 y 19 años no utilizan métodos anticonceptivos. Estudios previos (Canto, 2012; Manlove y otros, 2006) establecen una asociación entre los embarazos no deseados a corta edad, el riesgo de tener relaciones no protegidas y el poseer parejas considerablemente mayores. Sin embargo, Jenkins, Forero y Hembling (2014) reportan que no encontraron una asociación del uso de métodos anticonceptivos con el grado de escolaridad, el inicio temprano de la actividad sexual o tener más de dos parejas sexuales.

Con respecto a la escolaridad los resultados obtenidos en la investigación aportan evidencia para la hipótesis de que el embarazo adolescente representa un obstáculo importante para continuar los estudios: Dentro de la población evaluada, un 63.3\% tuvo que abandonar la escuela.

Por último la mayoría de las adolescentes tienen percepciones negativas en cuanto a las opiniones de sus familiares con respecto a su embarazo y relación de pareja. Sin embargo, dichas adolescentes expresan sentimientos positivos con respecto a ser madres y su relación de pareja. Pudimos encontrar que las preocupaciones más comunes entre las adolescentes estaban relacionadas con la incapacidad de continuar sus estudios la corta edad en que se encuentran y la diferencia de edad entre ellas y sus parejas.

Dentro de la normalidad durante la adolescencia se debe desarrollar un pensamiento operativo formal que debe permitir al adolescente profundizar y analizar sobre distintos factores que envuelven su vida (Fonagy, Gergely, Jurist, \& Target, 2004), proponen que este pensamiento es necesario para analizar y observar con más complejidad sus relaciones con las figuras de apego lo cual aporta a la toma de 
decisiones sobre ellas. Con relación a esto encontramos que muchas de las respuestas proporcionadas por las adolescentes eran dicotómicas -bien o mal-o no sé y no eran acompañadas de una aclaración que permitiera inferir la existencia de un proceso de reflexión sobre ellas. Respecto a este tema se pueden tener dos perspectivas: primero es posible que el involucrarse en una relación con un hombre significativamente mayor y ser madre o futura mamá haya sido resultado de la interrupción del establecimiento de dicho pensamiento operativo formal y como consecuencia encontramos estos patrones de apego no sanos y distintas dificultades tanto familiares como personales. Esta formulación quizás no puede servir como explicación de los patrones establecidos por las adolescentes evaluadas en la actualidad pero funciona como un factor predictor de futuras relaciones dentro y fuera del seno familiar. Segundo se podría creer que las respuestas proporcionadas se debieran a resistencias que tuvieran las adolescentes a la hora de contestar las preguntas debido a lo sensible del tema.

\section{Referencias.}

Austin, K., Campo, Z., De León, R., de Aguilar, C., Grajales, B., Arango, L., y Sandoval, I. (2000). Embarazo en adolescentes: Características psicosociales importantes. Regiones de Salud Metropolitana y de San Miguelito. Panamá: Instituto de la Mujer de la Universidad de Panamá.

Bartholomew, K. y Horowitz, L.M. (1991). Attachment styles among young adults: a test of a fourcategory model. Journal or Personality and Social Psychology, 61(2), 226-244

Cueva, A., Olvera, F. y Chumacera, R. (2005). Características sociales y familiares de las adolescentes embarazadas atendidas en un módulo de alto riesgo. Revista Médica del Instituto Mexicano del Seguro Social, 43(3), 267-271.

Camacho Hubner, A. V. (2000). Perfil de salud sexualy reproductiva en adolescentes y jóvenes de América Latina y el Caribe: Revisión bibliográfica, 1988-1998. Washington, DC: Organización Panamericana de la Salud, División de Promoción y Protección de la Salud.

Canto, N. (2012). Modelo cultural implicado en las relaciones de género y su influencia en la desigualdad de poder en la salud sexual y reproductiva de los adolescentes de la Ciudad de Panamá. Societas, 14(1), 51-74.

Cyr, C., Euser, E. M., Bakermans-Kranenburg, M. J., y Van Ijzendoorn, M. H. (2010). Attachment security and disorganization in maltreating and high-risk families: A series of meta-analyses. Development and psychopathology, 22(01), 87-108.

De León, R., Martínez, L., Chu, E., Mendoza, A., Chamorro, F., Poveda, C., y Rodríguez, F. (2011). Encuesta Nacional de Salud Sexualy Reproductiva 2009. Panamá: Instituto Conmemorativo Gorgas de Estudios de la Salud. 
Invest. pens. crit.

Vol. 3, No. 2, mayo-agosto 2015

pp. $20-31$

Fonagy, P., Gergely, G., Jurist, E., y Target, M. (2004). Affect regulation, mentalization and the development of the self. Lóndres, Inglaterra: Karnac Books

Gómez, P. I., Molina, R., y Zamberlin, N. (2011). Factores relacionados con el embarazo y la maternidad en menores de 15 años en América Latina y el Caribe. Lima: Promsex

Hohmann-Marriott, B. (2009). The couple context of pregnancy and its effects on prenatal care and birth outcomen. Maternal and Child Journal, 13(6), 745-754.

Instituto Nacional de Estadística y Censo [INEC]. (2010). XI Censo Nacional de Población: Resultados finales básicos. Panamá: Contraloría General de la República.

Instituto Nacional de Estadística y Censo [INEC]. (2014). Encuesta de indicadores múltiples por conglomerado 2013. Panamá: Contraloría General de la República.

Jenkins, L., Forero, I., y Hembling, J. (2014). Factores que influyen en el uso de métodos anticonceptivos modernos en la población panameña sexualmente activa entre los 15-59 años de edad en el año 2009. Investigación y Pensamiento Crítico, 2(5), 51-62.

Kaye, D. K., Mirembe, F. M., Bantebya, G., Johansson, A., y Ekstrom, A. M. (2006). Domestic violence during pregnancy and risk of low birthweight and maternal complications: a prospective cohort study at Mulago Hospital, Uganda. Tropical Medicine y International Health, 11(10), 15761584.

Manlove, J., Terry-Humen, E., y Ikramullah, E. (2006). Young teenagers and older sexual partners: Correlates and consequences for males and females. Perspective on Sexual and Reproductive Health, 38(4), 197-207.

Melero, R., y Cantero, M. J. (2008). Los estilos afectivos en la población española: un cuestionario de evaluación del apego adulto. Clínica y Salud, 19(1), 83-100.

Miller, K. S., Clark, L. F., y Moore, J. S. (1997). Sexual initiation with older male partners and subsequent HIV risk behavior among female adolescents. Family Planning Perspective, 29(5), 212-214.

Organización Mundial de la Salud [OMS]. (2009). Embarazo en adolescentes: un problema culturalmente complejo. Boletín de la Organización Mundial de la Salud, 87, 485-564.

Organización Mundial de la Salud [OMS]. (2012). Prevenir el embarazo precozy los resultados reproductivos adversos en adolescentes en los países en desarrollo: Las evidencias. Suiza: Autor.

Peterson, C.H., Buser, T.J., y Westburg, N. (2010). Effects of familial attachment, social support, involvement and self-esteem on youth substance use and sexual risk taking. The Family Journal, 18(4), 269-276. 
Programa de las Naciones Unidas para el Desarrollo [PNUD]. (2014). Informe nacional de desarrollo bumano Panamá 2014. El futuro es ahora: Primera infancia, juventudy formación de capacidades para la vida. Panamá: Autor.

Rangel, J.L., Valerio, L., Patiño, J. y García, M. (2004). Funcionalidad familiar en la adolescente embarazada. Rev. Fac. Med. UNAM, 47(1), 24-27

Silverman, J.G., Gupta, J., Decker, M.R., Kapur, N., y Raj, A. (2007). Intimate partner violence and unwanted pregnancy, miscarriage, induced abortion, and stillbirth among a national sample of Bangladeshi women. BJOG, 114(10), 1246-52.

Simpson, W. A. (2007). Attachment and the experience and expression of emotions in romantic relationships: A developmental perspective. Journal of Personality and Social Psychology, 92(2), 355-367.

Stephenson, R., Koeing, M., Acharya, R., y Tarun, R. (2008). Domestic violence, contraceptive use, and unwanted pregnancy in rural India. Studies in Family Planing, 39(3), 177-186.

Varela, M. (2010). Atlas social: Situación de las madres adolescentes. Panamá: Ministerio de Economía y Finanzas.

Winnicott, D. (1960). Los procesos de maduración y el ambiente facilitador. Buenos Aires, Argentina: Paidós. 